\title{
Analysis of FHIT Gene Methylation in Egyptian Breast Cancer Women: Association with Clinicopathological Features
}

\author{
Seham Mahrous Zaki ${ }^{1}$, Hala A Abdel-Azeez ${ }^{1 \&}$, Mona Roshdy El Nagar ${ }^{2 \&}$, Khaled \\ Abdel-Aziz Metwally ${ }^{3 \&}$, Marwa M Samir S Ahmed ${ }^{1 *}$
}

\begin{abstract}
Background: Fragile histidine triad (FHIT) gene is a tumor suppressor gene which involved in breast cancer pathogenesis. Epigenetics alterations in FHIT contributes to tumorigenesis of breast cancer. Objective: Our objective was to study FHIT promoter region hypermethylation in Egyptian breast cancer patients and its association with clinicopathological features. Materials and Methods: Methylation-specific polymerase chain reaction was performed to study the hypermethylation of FHIT promoter region in 20 benign breast tissues and 30 breast cancer tissues. Results: The frequency of hypermethylation of FHIT promoter region was significantly increased in breast cancer patients compared to bengin breast disease patients. The Odd's ratio (95\% CI) of development of breast cancer in individuals with FHIT promoter hypermethylation (MM) was 11.0 (1.22-250.8). There were also significant associations between FHIT promoter hypermethylation and estrogen, progesterone receptors negativity, tumor stage and nodal involvment in breast cancer pateints. Conclusions: Our results support an association between FHIT promotor hypermethylation and development of breast cancer in Egyptian breast cancer patients. FHIT promoter hypermethylation is associated with some poor prognostic features of breast cancer.
\end{abstract}

Keywords: FHIT gene - hypermethylation - breast cancer

Asian Pac J Cancer Prev, 16 (3), 1235-1239

\section{Introduction}

Breast Cancer is the most commonly diagnosed cancer amongst women worldwide. There were approximately 1.38 million new cases of breast cancer in the year 2008 and by 2020 this figure is anticipated to escalate to 1.7 million (Bhikoo et al., 2011). In Egypt, it is representing $18.9 \%$ of total cancer cases among the Egypt National Cancer Institute's series of 10,556 patients during the year 2001 (Salem et al., 2010).

Although cancer is a disease driven by genetic abnormalities, recent research suggests that epigenetic alterations of gene function are also central to the pathogenesis of these diseases. Specifically, abnormal promoter region methylation in candidate tumorsuppressor genes contributes to tightly heritable gene silencing and can, thereby, cause loss of gene function, which contributes to tumorigenesis of epithelial cancers. The spectrum of genes involved suggests that specific tumors may have their own distinct pattern of methylation (Wali , 2010).

The FHIT gene is located at chromosome 3p14.2. It spans $1.8 \mathrm{Mb}$ and has 10 exons. (Golebiowski et al., 2004). The chromosomal localization of FHIT in the common fragile region of the human genome suggests a positive correlation between the loss or inactivation of the FHIT gene and carcinogenesis (Haroun et al., 2014). Over the years, strong evidence has developed to support the postulation that FHIT is a tumor-suppressor gene (Wali, 2010). As predicted for a tumor suppressor, the Fhit protein is absent or markedly reduced in most human cancers (Haroun et al., 2014). In addition to homozygous deletion, FHIT gene structure is also subjected to (loss of heterozygosity) $\mathrm{LOH}$ and promoter hypermethylation (Choi et al., 2007). Silencing of the FHIT gene by promoter hypermethylation occurs in a number of cancers, such as bladder cancer (Han et al., 2011), nasopharyngeal carcinoma (Chen et al., 2013), lung cancers (Tan et al., 2013; Haroun et al., 2014; Li et al., 2014) and breast carcinomas (Syeed et al., 2011; Jeong et al., 2013).

Our objective was to study FHIT promoter region hypermethylation in Egyptian breast cancer patients and its association with clinicopathological features.

\section{Materials and Methods}

The study was performed at Clinical Pathology, General Surgery and Pathology Departments, Zagazig University Hospitals on 30 primary breast cancer and 20 age matched benign breast diseases women. Breast tissues 
were collected at Surgery Department after obtaining appropriate Institutional Review Board permission and informed consent from the patients. Benign breast tissues were obtained from women undergoing breast mass resection for biopsy. Primary breast tumors were obtained from women undergoing modified radical mastectomy. Only histopathologically confirmed benign and tumor tissues were included in the study. Tissues were collected directly into sterile collection vials containing chilled PBS (PH 7.2) and were stored at $-80^{\circ} \mathrm{C}$ until further analysis. The tumor stage and tumor size were classified according to Tumor Node Metastasis (TNM) staging system for breast cancer (Singletary and Connolly., 2006). The tumor histological types and tumor grading were classified according to World Health Organization (WHO) classification of breast tumor. Age, menopausal status and CA15-3 of studied patients are shown in Table 1.

Methylation-specific polymerase chain reaction (MSP) Genomic DNA was prepared from tissue samples by DNA preparation kit (Jena Bioscience, Germany). Bisulfite modification of genomic DNA was performed using Epimark Bisulfite Conversion Kit (New England Biolabs Inc,USA). Treatment of genomic DNA with sodium bisulfite converts unmethylated but not methylated cytosines to uracil, which are then converted to thymidine by alternating cycles of thermal denaturation with incubation reaction, producing sequence differences between methylated and unmethylated DNA (Frommer et al., 1992).

Primer sequences for the methylated FHIT reaction were 5’ TTG GGG CGC GGG TTT GGG TTT TTA CGC 3' (forward) and 5' CGT AAA CGA CGC CGA CCC CAC TA 3' (reverse), and for the unmethylated FHIT reaction were 5' TTG GGG TGT GGG TTT GGG TTT TTA TG 3' (forward) and 5' CAT AAA CAA CAC CAA CCC CAC TA 3’ (reverse) (Zöchbauer-Müller et al., 2001). PCR was done by 2X Hot Start PCR Master Mix (Tiangen Biotech,

Table 1. Age, Menopausal Status and CA15-3 Levels in Studied Patients

\begin{tabular}{lccc}
\hline Varaibles & $\begin{array}{c}\text { Benign Breast } \\
\text { Disease }(\mathrm{N}=20)\end{array}$ & $\begin{array}{c}\text { Breast Cancer } \\
(\mathrm{N}=30)\end{array}$ & $\mathrm{p}$ \\
\hline Age (years) & $42.1 \pm 9.1$ & $45.4 \pm 10.0$ & 0.22 \\
Menopausal status & & & \\
$\quad$ Pre menopausal & $18(90)$ & $18(60)$ & $0.02^{\mathrm{a}}$ \\
Post menopausal & $2(10)$ & $12(40)$ & \\
CA15-3(U/ml) & 15.4 & 37.8 & $<0.001^{\mathrm{b}}$ \\
& $(4.7-29.5)$ & $(12-1118)$ & \\
\hline
\end{tabular}

*Significant; $\mathrm{N}$ :nunmber of subjects; Data are expressed as number (\%), mean $\pm \mathrm{SD}^{\mathrm{a}}$ or median (range) ${ }^{\mathrm{b}}$

Table 2. FHIT Promoter Region Methylation Status in Both Studied Groups

\begin{tabular}{lccc}
\hline Varaibles & $\begin{array}{c}\text { Benign Breast } \\
\text { Disease }(\mathrm{N}=20)\end{array}$ & $\begin{array}{c}\text { Breast Cancer } \\
(\mathrm{N}=30)\end{array}$ & $\mathrm{p}$ \\
\hline Unmethylated (UU) & $15(75)$ & $9(30)$ & $0.004^{*}$ \\
Methylated (MM) & $1(5)$ & $11(36.7)$ & \\
Methylated/ & $4(20)$ & $10(33.3)$ & \\
Unmethylated (MU) & & & \\
\hline
\end{tabular}

*Significant; N:nunmber of subjects; Data are expressed as number (\%)
Beijing), using a total volume of $25 \mu 1$ (12.5 $\mu 1$ of Hot Start Master Mix with Loading Dye, $1 \mu 1$ of each primers, $5 \mu 1$ of modified bisulfate DNA,5.5 1 of dd $\mathrm{H} 2 \mathrm{O}$ ). Amplification was started using thermocycler (Gene Ampi 2400 PCR system) as follow: preheat at $94^{\circ} \mathrm{C}$ for $3 \mathrm{~min}$, followed by 40 cycles $\left(95^{\circ} \mathrm{C}\right.$ for $30 \mathrm{sec}, 65^{\circ} \mathrm{C}$ for $30 \mathrm{sec}, 72^{\circ} \mathrm{C}$ for $\left.30 \mathrm{sec}\right)$ and final extension at $72^{\circ} \mathrm{C}$ for $7 \mathrm{~min}$ (Zheng et al., 2004). Negative control samples without DNA were included for each set of PCR . The PCR products were separated on $1.8 \%$ agarose gels, stained using ethidium bromide and visualized under UV illumination. The PCR generated a 74bp products for both methylated and unmethylated DNA are shown in Figure 1 and 2.

\section{Statistical analysis}

Data were checked, entered and analysed by using SPSS vesion 20(Chicago,IL,USA). Data were expressed as number and percentage for categorical variables, mean \pm SD or median and range for quantitative variables. Chi-square $\left(\mathrm{X}^{2}\right), \mathrm{t}$ test, and Mann whitnny were used for groups comparison when appropriate. Odd's ratio and 95 $\%$ confidence interval $(\mathrm{CI})$ was performed to predict the effect of hypermethylation of FHIT gene on development of breast cancer. $\mathrm{p}<0.05$ was considered statistically significant.

\section{Results}

FHIT promoter region hypermethylation status in

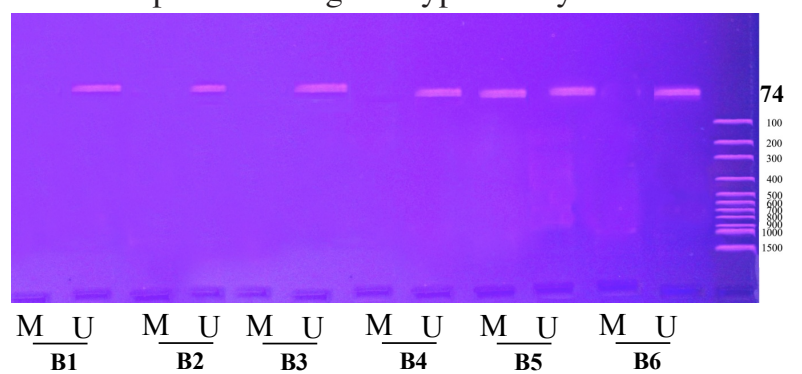

Figure 1. Methylation Status of FHIT Promoter Region (74bp) from 6 Benign Breast Tissue Samples. Lanes marked M: Methylated, lanes marked U: unmethylated, Lane marked L: 100bp molecular ladder. B1,2,3,4,6 represent unmethylated FHIT promoter region (UU) and B5 represents methylated,unmethylated FHIT promoter region (MU)

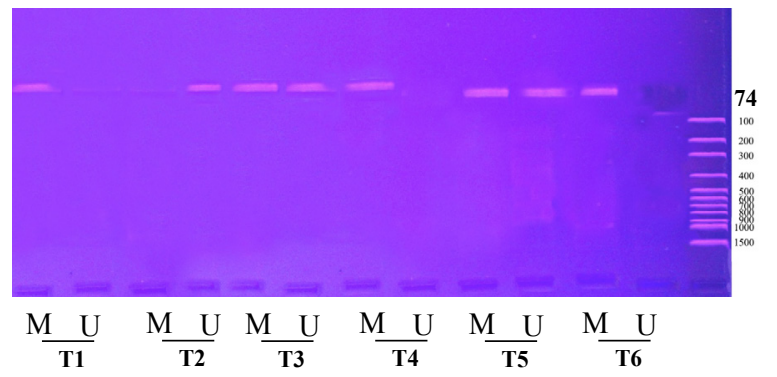

Figure 2. Methylation Status of FHIT Promoter Region (74bp) from 6 Breast Cancer Tissue Samples. Lanes marked M: Methylated, lanes marked U: unmethylated, Lane marked L: 100bp molecular ladder. T1,4,6 represent methylated FHIT promoter region (MM),T3,5 represent methylated, unmethylated FHIT promoter region (MU)andT2 represents unmethylated FHIT promoter region (UU) 
benign and cancer breast tissues is shown in Table 2 . There was significant increase in FHIT methylation in breast cancer patients compared to benign breast disease patients $(\mathrm{p}=0.004)$. Odd's ratio $(95 \% \mathrm{CI})$ of development of breast cancer in individuals with FHIT promoter hypermethylation was $11.0(1.22-250.8, \mathrm{p}=0.016)$ in $\mathrm{MM}$, was $2(0.45-9.4, \mathrm{p}=0.3)$ in $\mathrm{MU}$, and it was $0.14(0.03-0.6$, $\mathrm{p}=0.0018)$ in UU gene. Both MM, MU were considered as FHIT metylation positive (FHIT +ve) and UU as FHIT metylation negative (FHIT-ve).

The relationships between FHIT gene hypermethylation status and clinicopathological features in breast cancer patients were evaluated. There was significant association between FHIT promoter region hypermethylation and hormonal receptors negativity (ER,PR), lymph node involvement and tumor stage while no significant association between FHIT promoter region hypermethylation and menopausal status, tumor size, tumor grade, histological types and CA15-3 ( $\mathrm{p}>0.05)$ (Table 3).

Table 3. Association between FHIT Hypermethylation and Clinicopathological Features

\begin{tabular}{|c|c|c|c|}
\hline Varaibles & $\begin{array}{c}\text { FHIT -ve } \\
\text { N=9 }\end{array}$ & $\begin{array}{c}\text { FHIT +ve } \\
\quad \mathrm{N}=21\end{array}$ & $\mathrm{p}$ \\
\hline \multicolumn{4}{|l|}{ Menopausal Status } \\
\hline Premenopausal & $4(44.4)$ & $14(66.7)$ & 0.46 \\
\hline Postmenopausal & $5(55.6)$ & $7(33.3)$ & \\
\hline \multicolumn{4}{|l|}{ Tumor size } \\
\hline $\mathrm{T}_{1}$ & $3(33.3)$ & $1(4.76)$ & 0.14 \\
\hline $\mathrm{T}_{2}$ & $4(44.4)$ & $9(42.9)$ & \\
\hline $\mathrm{T}_{3}$ & $1(11.1)$ & $8(38.1)$ & \\
\hline $\mathrm{T}_{4}$ & $1(11.1)$ & $3(14.3)$ & \\
\hline \multicolumn{4}{|l|}{ Tumor grade } \\
\hline I & $1(11.1)$ & $1(4.76)$ & 0.51 \\
\hline II & $6(66.7)$ & $11(52.4)$ & \\
\hline III & $2(22.2)$ & $9(42.9)$ & \\
\hline \multicolumn{4}{|l|}{ Tumor stage } \\
\hline I & $4(44.4)$ & $0(0.0)$ & $0.003^{*}$ \\
\hline II & $5(55.5)$ & $11(52.4)$ & \\
\hline III & $0(0.0)$ & $6(28.6)$ & \\
\hline IV & $0(0.0)$ & $4(19.0)$ & \\
\hline \multicolumn{4}{|l|}{ LN involvment } \\
\hline Postive & $6(66.7)$ & $20(95.5)$ & $0.03^{*}$ \\
\hline Negative & $3(33.3)$ & $1(4.76)$ & \\
\hline \multicolumn{4}{|l|}{ Metastasis } \\
\hline M0 & $8(88.9)$ & $18(85.7)$ & 0.74 \\
\hline M1 & $1(11.1)$ & $3(14.3)$ & \\
\hline \multicolumn{4}{|l|}{ Histological types } \\
\hline IDL & $6(66.7)$ & $18(85.7)$ & 0.24 \\
\hline ILC & $2(22.2)$ & $1(4.76)$ & \\
\hline Others & $1(11.1)$ & $2(9.52)$ & \\
\hline \multicolumn{4}{|l|}{ ER status } \\
\hline Postive & $6(66.7)$ & $4(19.0)$ & 0.012 \\
\hline Negative & $3(33.3)$ & $17(81.0)$ & \\
\hline \multicolumn{4}{|l|}{ PR status } \\
\hline Postive & $6(66.7)$ & $4(19.0)$ & 0.012 \\
\hline Negative & $3(33.3)$ & $17(81.0)$ & \\
\hline CA15-3 (U/ml) ${ }^{\mathrm{a}}$ & $\begin{array}{l}28 \\
(15.6-250)\end{array}$ & $\begin{array}{l}40 \\
(12-1118)\end{array}$ & 0.25 \\
\hline
\end{tabular}

*significant; LN : Lymph node; IDC : Intraductal cancer; ILC:Intralobular cancer; ER : estrogen receptor; PR:progesterone receptor; Data are expressed as number (\%) or median (range) ${ }^{\mathrm{a}}$

\section{Discussion}

Considerable variations in promoter methylation in individual genes existing in the profiles of different cancers could be molecular markers, which would be capable of distinguishing among the various individual tumors and also their normal counterparts (Esteller, 2003; Laird, 2003; Patel et al., 2003). We studied the methylation status of the 5' CpG island of FHIT promotor in benign breast diseases and breast cancers women. Our study revealed significant increase in hypermethylation status in breast cancer patients compared to benign breast diseases ( $\mathrm{p}=0.004)$. Our results were similar to previously reported studies (Roa et al., 2004; Raish et al., 2009). A recent quantative analysis of FHIT promoter hypermethylation in primary breast cancer by using pyrosequencing showed high percentage of FHIT methylation $(96.7 \%)$ in breast cancer patients (Jeong et al., 2013). Promoter aberrant methylation of FHIT is an important mechanism for inactivation of this tumor suppressor gene in mammary tumorigenesis (Syeed et al., 2011).

Major progress in controlling mortality and morbidity from cancer requires better understanding of molecular mechanisms underlying disease initiation. Our study revealed hypermethylation in breast tissues obtained from some patients with benign breast diseases (25\%) that might represent the appearance of premalignant lesions in those patients. FHIT promoter hypermethylation was associated with increased risk of development of breast cancer in benign breast diseases patients (Odd's ratio was 11.0, $\mathrm{p}=0.016$ in MM gene). Analysis of early aberrant events is complex because by the time the tumor is detected, the cancer progenitor cells may have already undergone multiple changes both at genetic and epigenetic levels (Raish et al., 2009). Detection of FHIT hypermethylation using MSP might provide potential new molecular diagnostic markers of breast carcinomas at an early stage during multistep carcinogenesis (Syeed et al., 2011).

On the other hand, a significant proportion of breast cancer patients were found to have normal FHIT gene without any apparent methylation (Gatalica et al., 2000; Raish et al., 2009). This indicates that routes independent of this gene may be involved in the pathogenesis of this disease (Rasih et al., 2009). The variation between our results and these results could be attributed to the small sample tissue size, the proportion of cancerous cells and ethinic differences.

FHIT Gene is a target of breast cancer-specific chromosome 3p alterations (Yang et al., 2002). Notably, the rate of hypermethylation at FHIT is higher than the percentage of $\mathrm{LOH}$ at the FHIT locus, which suggests that FHIT hypermethylation is a more common event in breast carcinoma. Biallelic inactivation of the FHIT gene could result from epigenetic inactivation of both parental alleles and could be reversed by exposure to demethylating agents (Zochbauer-Muller et al., 2001). It is tempting to speculate that demethylating agents might have a role in cancer prevention for individuals who are at risk for cancer or for individuals in whom FHIT promoter hypermethylation is detected as an early neoplastic change. Moreover, knowledge of the FHIT hypermethylation state in primary 
breast cancers may be useful to identify tumors that are more likely to respond to FHIT-demethylating therapy (Yang et al., 2002).

We also evaluated the association between FHIT gene hypermethylation and breast cancer clinicopathologic characteristics. FHIT gene hypermethylation was found to be significantly associated with estrogen and progesterone receptors negativity $(\mathrm{p}=0.012)$. Our results were in agreement with Yang et al. (2001) who found association between FHIT hypermethylation and ER negativity. Rasih et al. (2009) reported significant associations between FHIT hypermethylation and ER and PR negativity among postmenopausal women, while in premenopausal women FHIT hypermethylation was more frequent among ER and PR postive patients. Other studies have found that FHIT hypermethylation is more strongly associated with PR than with ER and that PR negativity might have a stronger prognostic role than ER in patients with breast cancer (Arun et al., 2005; Naqvi et al., 2008). When hormonal receptors negativity combined with the methylation status, these patients have the potential to respond differently to the various treatment options currently available (Raish et al., 2009). Methylation in breast cancer has long been linked to the hormone regulation, but this correlation is not intelligible yet. DNA methylation profiles in breast, endometrial, ovarian, and proximal colon cancers provide contradictory evidence for global hormone-specific DNA methylation signatures (Campan et al., 2006).

Our study revealed significant association between methylation of FHIT gene and breast cancer stages progression $(\mathrm{p}=0.003)$, thereby suggesting that FHIT gene silencing due to methylation is involved in the progression of breast tumorigenesis in breast cancer women. Our results were found to be comparable with the findings of other workers (Zochbauer-Muller et al., 2001; Terry et al., 2007; Naqvi et al., 2008; Syeed et al., 2011), but different from those described by Kim et al. (2006). Haroun et al. (2014) reported also association between FHIT methylation and lung cancer's later metastatic stage, the study suggested that methylation of FHIT is a useful biomarker of biologically aggressive disease in patients with non small cell lung cancer.

We found a significant association between FHIT gene hypermethylation and nodal involvment. FHIT gene hypermethylation was more among nodal positive cases $(\mathrm{p}=0.03)$. This result was in agreement with Syeed et al. (2011) study.

In this study, we didn't find significant association between FHIT gene hypermethylation and other clinicopathological features namely menopausal status , tumor size, histological type, presence of metastasis and tumor grade. Previous studies didn't report association of FHIT promotor hypermethylation and tumor size, histological types and tumor grade (Zochbauer-Muller et al., 2001; Iliopoulos et al., 2005; Syeed et al., 2011; Jeong et al., 2013). While other studies found association between FHIT hypermethylation and poorly differentiated breast cancer patients (yang et al., 2001; Li et al., 2006; Tao et al., 2009; Rasih et al., 2009), their finding is important because it links DNA hypermethylation with the histologic appearance of breast cancers (Rasih et al., 2009). Our finding concerning the non significant association between FHIT gene hypermethylation and menopausal status was different from a previous study who reported an association between FHIT gene hypermethylation and pre-menopausal women and found that the premenopausal women were at approximately twice risk, than the postmenopausal women (Syeed et al., 2011).

In conclusion, our results support the association between FHIT promoter hypermethylation and development of breast cancer. FHIT promoter hypermethylation is associated with some poor prognostic features of breast cancer. It has the potential to be used clinically in diagnosis, prognosis and recurrence evaluation in those patients. The clinical significance of our findings should be further evaluated in larger studies with follow-up to evaluate their effect on disease-free and overall survival.

\section{References}

Arun B, Kilic G, Yen C, et al (2005). Loss of FHIT expression in breast cancer is correlated with poor prognostic markers. Cancer Epidemiol Biomarkers Prev, 14, 1681-5.

Bhikoo R, Srinivasa S, Yu TC, et al (2011). Systematic review of breast cancer biology in developing countries (part 1): Africa, the Middle East, Eastern Europe, Mexico, the Caribbean and South America. Cancers, 3, 2358-81.

Campan M, Weisenberger DJ, and Laird PW (2006). DNA methylation profiles of female steroid hormone-driven human malignancies. Curr Top Microbiol Immunol, 310, 141-78.

Chen X, Li P, Yang Z, Mo WN (2013). Expression of fragile histidine triad (FHIT) and WW-domain oxidoreductase gene (WWOX) in nasopharyngeal carcinoma. Asian Pac J Cancer Prev, 14, 165-71.

Choi CH, Lee KM, Choi JJ, et al (2007). Hypermethylation and loss of heterozygosity of tumor suppressor genes on chromosome 3p in cervical cancer. Cancer Lett, 255, 26-33.

Esteller M (2003). Relevance of DNA methylation in the management of cancer. Lancet Oncol, 4, 351-8.

Frommer M, Mcdonld LE, Millar DS, et al (1992). A genomic sequencing protocol that yields a positive display of 5-methylcytosine residues in individual DNA strands. Proc Natl Acad Sci USA, 89, 1827-31.

Gatalica Z, Lele SM, Rampy BA, and Norris BA (2000). The expression of Fhit protein is related inversely to disease progression in patients with breast carcinoma. Cancer, $\mathbf{8 8 ,}$ 1378-83.

Golebiowski F, Szulc A, Szutowicz A, Pawelczyk T (2004). Ubc9-induced $\mathrm{V}$ inhibition of diadenosine triphosphate hydrolase activity of the putative; tumor suppressor protein FHIT. Arch Biochem Biophy, 428, 160-4.

Han Y, Zhang Z, Zhang GJ, et al (2011). Aberrant FHIT expression is linked to bladder carcinogenesis and apoptosis. Asian Pac J Cancer Prev, 12, 2915-20.

Haroun RA, Zakhary NI, Mohamed MR, et al (2014). Assessment of the prognostic value of methylation status and expression levels of FHIT, GSTP1 and p16 in non-small cell lung cancer in Egyptian patients. Asian Pac J Cancer Prev, 15, 4281-7.

IIiopoulos D, Guler G, Han SY, et al (2006). Roles of FHIT and WWOX fragile genes in cancer. Cancer Lett, 232, 27-36

Jing F, Yuping W, Yong C, et al (2010). CpG island methylator phenotype of multigene in serum of sporadic breast carcinoma. Tumor Biol, 31, 321-31.

Jeong YJ, Jeong HY, Lee SM, et al (2013). Promotor methylation 
status of FHIT gene and Fhit expression: Association with HER2/neu status in breast cancer patients. Oncol Rep, 30, 2270-8.

Kim JS, Kim JW, Han J, et al (2006). Cohypermethylation of p16 and FHIT promoters as a prognostic factor of recurrence in surgically resected stage I non-small cell lung cancer. Cancer Res, 15, 4049-54.

Laird PW (2003). The power and the promise of DNA methylation markers. Nat Rev Cancer, 3, 253-66.

Li W, Deng J, Tang JX (2014). Combined effects methylation of FHIT, RASSF1A and RAR $\beta$ genes on non-small cell lung cancer in the Chinese population. Asian Pac J Cancer Prev, 15, 5233-7.

Li S, Rong M, Iacopetta B (2006). DNA hypermethylation in breast cancer and its association with clinicopathological features. Cancer Lett, 237, 272-80.

Naqvi RA, Hussain A, Raish M, et al (2008). Specific 5'CpG island methylation signatures of FHIT and p16 genes and their potential diagnostic relevance in Indian breast cancer patients. DNA Cell Biol, 27, 517-25.

Patel A, Groopman JD, Umar A (2003). DNA methylation as a cancer-specific biomarker: from molecules to populations. Ann NY Acad Sci, 98, 286-97.

Raish M, Dhillon VS, Ahmad A, et al (2009). Promoter hypermethylation in tumor suppressing genes p16 and FHIT and their relationship with estrogen receptor and progesterone receptor status in breast cancer patients from Northern India. Transl Oncol, 4, 264-70.

Roa JC, Anabalon L, Tapia O, et al (2004). Promoter methylation profile in breast cancer. Rev Med Chile, 13, 1069-77.

Salem AAS, Salem MA, Abbass H (2010). Breast cancer: surgery at the South Egypt cancer institute. Cancers, 2, 1771-8.

Singletary SE, Connolly JL (2006). Breast cancer staging:working with the sixth edition of the AJCC cancer staging manual. CA Cancer J Clin, 56, 37-47.

Syeed N, Husain SA, Sameer AS, Chowdhri NA, Siddiqi MA (2011). Mutational and promoter hypermethylation status of FHIT gene in breast cancer patients of Kashmir. Mutat Res, 707, 1-8.

Tan S, Sun C, Wei X, et al (2013). Quantitative assessment of lung cancer associated with genes methylation in the peripheral blood. Exp Lung Res, 39, 182-90.

Tao MH, Shields PG, Nie J, et al (2009). DNA hypermethylation and clinicopathological features in breast cancer: the Western New York Exposures and Breast Cancer (WEB) Study. Breast Cancer Res Treat, 114, 559-68.

Terry G, Ho L, Londesborough P, et al (2007). The expression of FHIT, PCNA and EGFR in benign and malignant breast lesions. Br J Cancer, 96, 110-7.

Wali A (2010). FHIT: doubts are clear now mini-review. Sci World $J, 10,1142-51$.

Yang Q, Yoshimura G, Suzuma T, et al (2001). Clinicopathological significance of fragile histidine triad transcription protein expression in breast carcinoma. Clin Cancer Res, 7, 3869-73.

Yang Q, Mori I, Shan L, et al (2001). Biallelic inactivation of retinoic acid receptor 2 gene by epigenetic change in breast cancer. Am J Pathol, 158, 299-303.

Yang Q, Yoshimura G, Sakurai T, Kakudo K (2002). The fragile histidine triad gene and breast cancer. Med Sci Monit, 8 , 140-4.

Zochbauer-Muller S, Fong KM, Maitra A, et al (2001). 5'CpG island methylation of the FHIT gene is correlated with loss of gene expression in lung and breast cancer. Cancer Res, 61, 3581-5.

Zheng S, Ma X, Zhang L, et al (2004). Hypermethylation of the 5_CpG Island of the FHIT Gene is associated with hyperdiploid and translocation-negative subtypes of pediatric leukemia. Cancer Res, 64, 2000-6. 